\title{
A Case of Pulmonary Embolism with Sarcomatoid Malignant Pleural Mesothelioma with Long-Term Pleural Effusion
}

\author{
Rumeng $\mathrm{Gu}^{1,2, *}$ \\ Luxi Jiang ${ }^{l, *}$ \\ Ting Duan' \\ Chun Chen' \\ Shengchang $\mathrm{Wu}^{\prime}$ \\ Deguang $M u^{\prime}$
}

'Department of Respiratory Medicine, Zhejiang Provincial People's Hospital, Affiliated People's Hospital, Hangzhou Medical College, Hangzhou, Zhejiang, People's Republic of China; ${ }^{2}$ Graduate School of Clinical Medicine, Bengbu

Medical College, Bengbu, People's

Republic of China

*These authors contributed equally to this work
Correspondence: Shengchang Wu;

Deguang $\mathrm{Mu}$

Tel +86-I5869I72806; +86-57I-

58593509

Email wushengchang@I26.com;

mudeguang@|26.com
Background: Malignant pleural mesothelioma (MPM) is a highly aggressive tumor that originates from pleural mesothelial cells. In recent years, with the development of asbestosrelated industries and the increase in air pollution, its incidence has been increased. The incidence of pulmonary embolism combined with sarcomatoid MPM is very low and the prognosis is extremely poor. We here report a case of a patient with long term of pleural effusion and finally diagnosed as pulmonary embolism with sarcomatoid MPM.

Case: A 75-year-old male with a 30-year history of asbestos exposure was admitted to our hospital due to chest pain and difficulty in breathing after exercise. Radiologic examination revealed pleural effusion, computed tomography pulmonary angiography (CTPA) suggests pulmonary embolism, and we consider pleural effusion caused by pulmonary embolism. After anticoagulant therapy for pulmonary embolism and pleural puncture to reduce pleural effusion, the patient's symptoms improved. However, after that, the patient was still admitted to the hospital several times because of recurrent chest pain and dyspnea symptoms, and radiologic examination always showed unexplained pleural effusion. Finally, pathological and immunohistochemical examinations of the pleural biopsy specimens were performed, and the diagnosis was confirmed as sarcomatoid MPM.

Conclusion: In summary, sarcomatoid MPM with pulmonary embolism is relatively rare, and the prognosis is poor. Clinicians need to be alert to its occurrence. When the first diagnosis is confirmed and the effect of targeted treatment is still not good, the possibility of other diseases should be considered. In clinical practice, pleural biopsy guided by PET-CT is a good choice for patients with sarcomatoid MPM who cannot tolerate open pleural biopsies or thoracoscopy. And patients should undergo pleural morphology and immunohistochemistry as soon as possible, which are helpful for timely diagnosis.

Keywords: malignant pleural mesothelioma, pleural effusion, asbestos

\section{Introduction}

Malignant pleural mesothelioma (MPM) is a rare and aggressive tumor. The prognosis is poor, with a median survival time ranging from 8 to 14 months. ${ }^{1}$ Most cases of MPM are associated with a history of asbestos exposure, with an incubation period of 10 to 50 years or more. ${ }^{2}$ And chrysotile, crocidolite or amosite asbestos are the three main types of asbestos that cause MPM. Occupational asbestos exposure is the most common, such as building construction workers, power station workers, electrical workers and boilermaker. ${ }^{2}$ There are three main histological subtypes of MPM: biphasic, epithelioid and sarcomatoid. Among them, the sarcomatoid variant has the worst prognosis, with 
a median survival time of only 4 months. ${ }^{1,3}$ There is a strong association between cancer and thrombosis. Studies have shown that 1 in 7 hospitalized cancer patients dies from pulmonary embolism. ${ }^{4}$ However, the incidence of sarcomatoid MPM combined with pulmonary embolism is extremely low, and the prognosis is poor. The imaging findings of MPM are mostly unilateral pleural effusion, pleural mass or pleural nodules. In the early stages of the disease, $70 \%$ of patients will find pleural effusion. ${ }^{5}$ Pulmonary embolism can also cause pleural effusion. Here, we report a case of pulmonary embolism with sarcomatoid MPM with long-term pleural effusion.

\section{Case Report}

A 75-year-old male patient was admitted to Zhejiang Provincial People's Hospital on 23 November 2018 after two-week-history of post-exercise dyspnea and chest pain. Physical examination revealed decreased breath sound, increased tactile fremitus on the affected side, and dullness to percussion of the left lung. Chest computed tomography (CT) showed diffuse thickening and calcification of the pleura on both sides, pleural effusion and atelectasis on the left side (Figure 1A and B). After hospitalization, the patient received a thoracentesis on November 26, 2018. The pleural fluid was bloody exudate, and no tumor cells were found in the exfoliated cells of the pleural fluid sample. According to the imaging findings, we tracked the patient's occupational history and learned that the patient had a history of close contact with asbestos for 30 years. The diagnosis of pleural mesothelioma needs to be ruled out clinically, and it is planned to be transferred to surgery for open pleural biopsy. At the same time, laboratory examination revealed elevated plasma D-dimer $(26190 \mathrm{Ug} / \mathrm{L})$, combined with the patient's chest pain, dyspnea symptoms, and a large amount of pleural

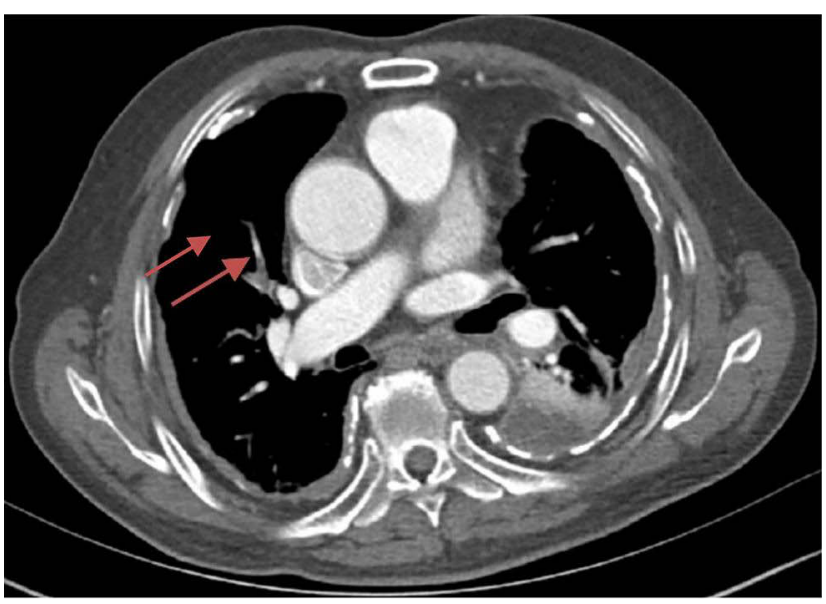

Figure 2 Right lung branch pulmonary embolism, mainly involved pulmonary artery (arrow).

effusion, the possibility of pulmonary embolism should be considered. Then, the computed tomography pulmonary angiography (CTPA) was performed for the patient, and the results showed that there was embolism in the right pulmonary artery branch (Figure 2). After the diagnosis of pulmonary embolism, we immediately used anticoagulant drugs for the patient. Because anticoagulants are easy to cause massive hemorrhage, no open pleural biopsy was performed. After anticoagulation therapy, the patient's symptoms of pleural effusion and dyspnea were improved and the patient discharged on December 18, 2018.

However, after being discharged from the hospital, the patient was still admitted to the hospital several times due to chest pain and dyspnea, and the reexamined chest CT still showed a large amount of pleural effusion on the left side (Figure 3A and B). When he was hospitalized again on July 8, 2019, there was no obvious abnormality in plasma D-dimer and CTPA, which ruled out the possibility of
A

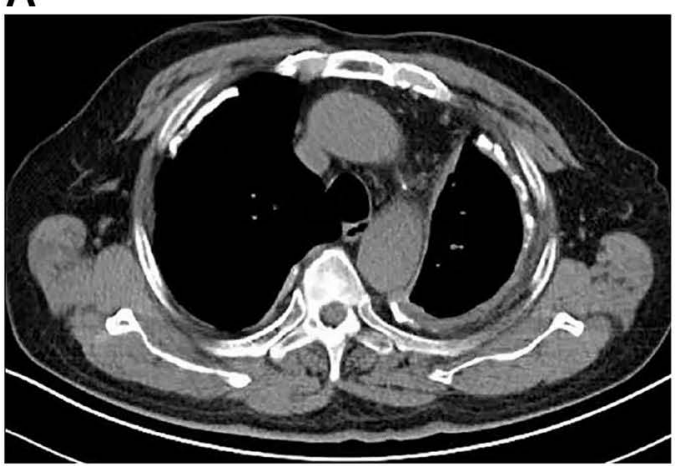

B

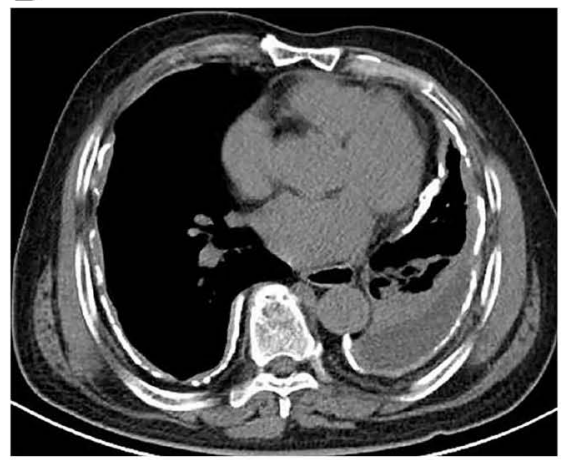

Figure I Mediastinal windows of computed tomography (A and $\mathbf{B})$ confirm the previously noted bilateral pleural thickening, calcification. There is associated left lobe Pleural effusion and atelectasis. 

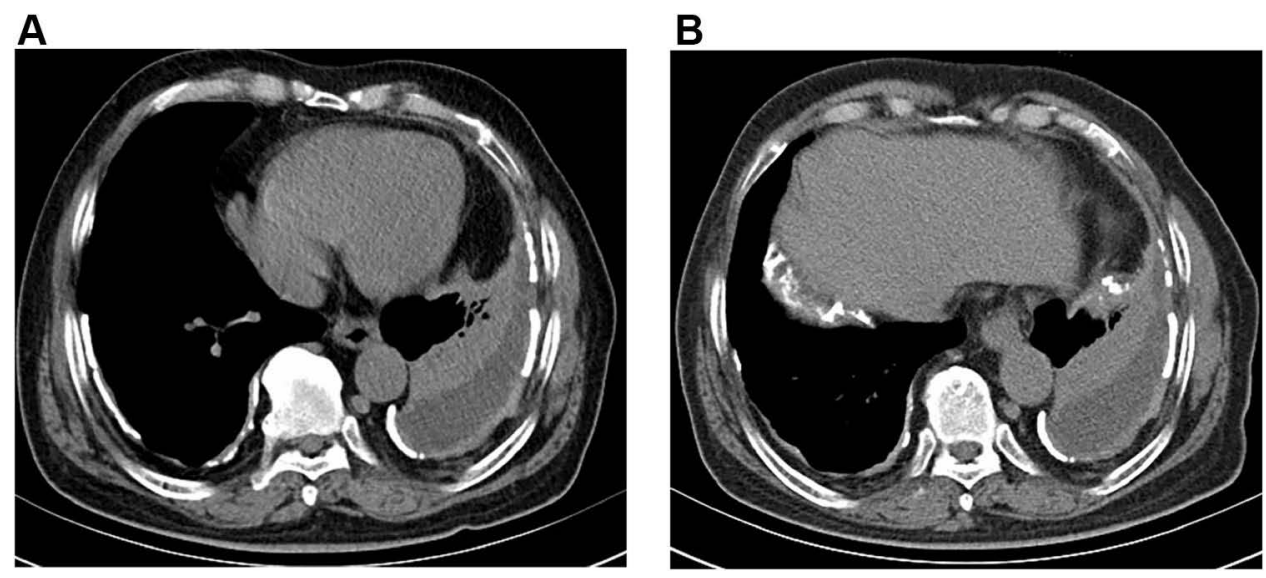

Figure 3 Mediastinal windows of computed tomography ( $\mathbf{A}$ and $\mathbf{B}$ ) revealed pleural effusion on the left side.

recurrent pulmonary embolism. We also considered the possibility of pneumonia and tuberculous pleurisy, but repeated pleural effusion and sputum culture did not show any positive results and there was no evidence for tuberculosis. Because of the long-term bloody pleural effusion, the possibility of lung cancer is also considered. The patient's pleural fluid was sent for pathological examination for many times, but no tumor cells were found. PET-CT examination showed diffuse thickening of the bilateral pleura with calcification, mainly on the left side. FDG metabolism was increased, and malignant tumors were considered (Figure 4). We consider that this patient may have malignant mesothelioma. Later, the patient underwent an ultrasound-guided pleural biopsy, and the pathological examination revealed fibroblast-like spindle cells arranged in bundles or chaotic shapes (Figure 5A and B). The results of immunohistochemistry were: $\mathrm{CK}(\operatorname{Pan})(+)$, WT1(+), GATA3(+), P63(-), TTF-1(-), EMA(-), CD117(-), DOG1 (-), Calretinin(-), CK5/6(-), CD34(-), ERG(-), SMA(-),

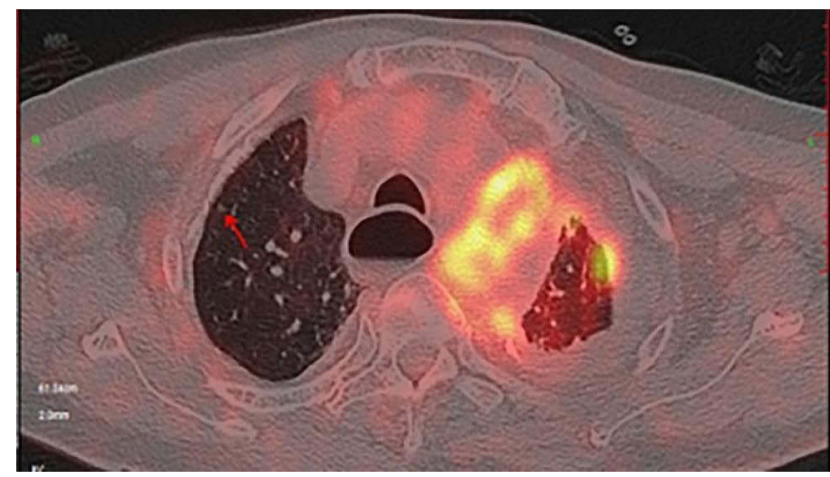

Figure 4 PET-CT suggests thickening and calcification of the pleura on both sides, increased FDG metabolism, more pronounced on the left.

Desmin(-), S100(-), MiTF(-), HMB45(Melanoma)(-), MyoD1(-), Caldesmon(-), D2-40(-), CEA(-), Vimentin(-) (Figure 6). Combined with the above examination results, the final diagnosis was sarcomatoid pleural mesothelioma. However, two days later, the patient suddenly developed a coma, facial cyanosis, and sudden cardiac arrest. And the
A

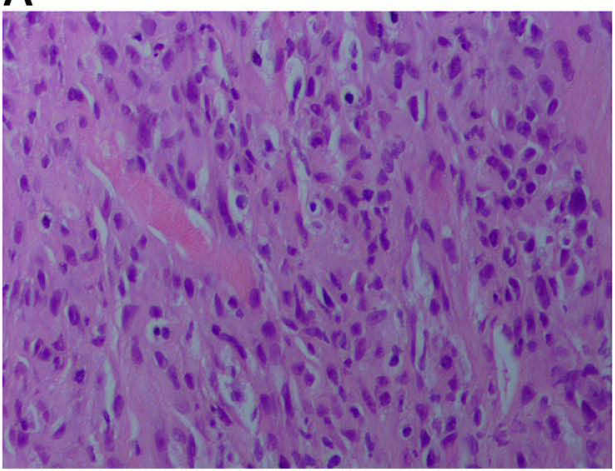

B

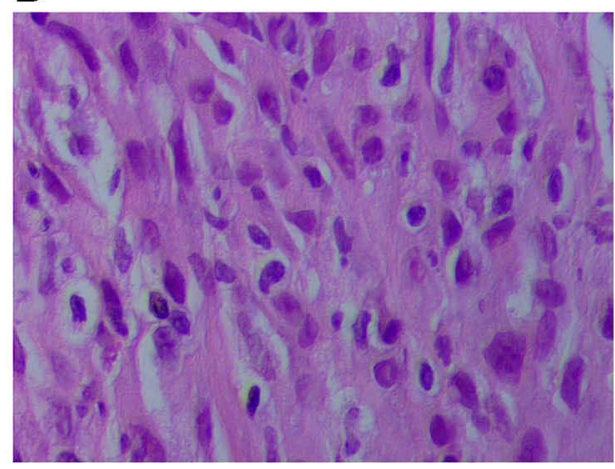

Figure 5 Ultrasound guided biopsy specimen from tumor lesions. Fibroblast-like spindle cells arranged in bundles or chaotically, the tumor cells had obvious atypia, mitotic figures, and coagulative necrosis. ((A and $\mathbf{B})$, hematoxylin-eosin). 

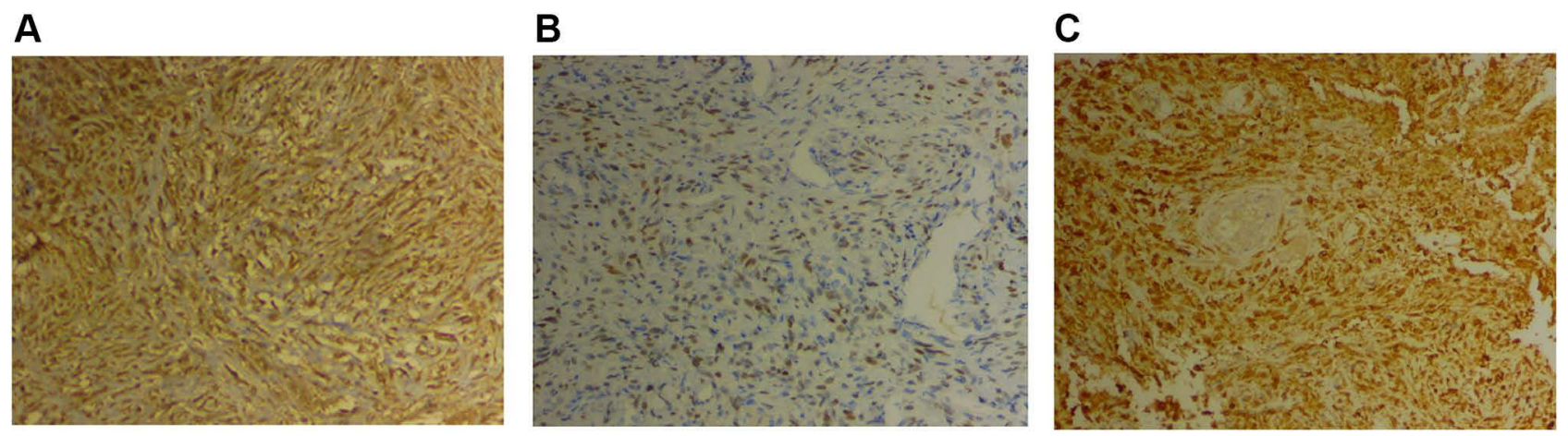

Figure 6 Immunohistochemical staining $(20$ X) revealed WT-I positivity (A), GATA-3 positivity (B) and CK (pan) positivity (C).

blood pressure, oxygen saturation cannot be measured at that time. We consider that the cause of cardiac arrest may be an acute myocardial infarction or acute pulmonary embolism. After tracheal intubation, electric defibrillation and chest compression, the patient discharged voluntarily. The patient died after being discharged from the hospital 2 days later.

\section{Discussion}

Malignant pleural mesothelioma (MPM) is a rare and almost universally fatal tumor. The Centers for Disease Control (CDC) reported 45,221 MPM-related deaths between 1999 and 2015, During this period, MPM mortality increased by $4.8 \%{ }^{6}$ The history of asbestos exposure is an important factor in the diagnosis of mesothelioma. Approximately $87.3 \%$ of male cases and $64.8 \%$ of female cases are caused by asbestos exposure, usually with an incubation period of several decades. ${ }^{7,8}$ In the past few decades, due to the increase in asbestos production and commercial use, the global incidence of MPM has been steadily rising. ${ }^{9-11}$ In the case we reported, we asked the patient's asbestos exposure history based on the patient's imaging studies, and considered that the patient might be diagnosed with MPM. Therefore, in the diagnosis of MPM, the history of asbestos exposure is particularly important. We must confirm the patient's occupational and exposure history as soon as possible.

Sarcomatoid MPM is relatively rare, accounting for about $10 \%$ of malignant mesothelial lesions, with poor prognosis and short survival. ${ }^{12}$ In 2015, WHO defined sarcomatoid malignant mesothelioma as the proliferation of spindle cells, which are mainly arranged in fascicles and can be divided into sarcomatoid mesothelioma, desmoplastic mesothelioma and heterogeneous mesothelioma tumor. ${ }^{12}$ However, morphological features are sometimes difficult to identify. It is necessary to perform immunohistochemical stains on paraffin block material.

The clinical symptoms of MPM are not specific, and are easy to be misdiagnosed. Most patients showed dyspnea and chest pain. Dyspnea is usually caused by pleural effusion. And chest pain may be caused by pleural effusion or tumor In our case, the patient had typical chest pain and dyspnea, which was consistent with MPM. However, the patient's CTPA suggested pulmonary embolism at that time, which explained the symptoms of chest pain, dyspnea, and pleural effusion. And the incidence of pleural effusion in patients with pulmonary embolism is $19.9 \%{ }^{13}$ Pleural mesothelioma with pulmonary embolism is rare. A study indicates that the incidence of pulmonary embolism in patients with pleural mesothelioma is $3.4 \% .{ }^{14}$ Mesothelioma cells secrete procoagulant factors and interleukin 6, which could enhance platelet function and thrombosis, and at the same time promote inhibition of fibrinolysis, leading to the formation of pulmonary embolism. ${ }^{15,16}$ In addition, due to the patient's anticoagulation therapy, considering the risk of bleeding during invasive procedures, no open pleural biopsy was performed. In fact, anticoagulation therapy is not an absolutely contraindication to perform diagnostic videoassisted thoracic surgery (VATS) and it could have been performed under local anesthesia and sedation. ${ }^{17,18}$ Therefore, MPM was not diagnosed in time at the first visit. Subsequently, the patient developed persistent pleural effusion that could not be attributed to the pulmonary embolism. Based on the poor effectiveness of the treatment for pulmonary embolism, we considered the possibility of other diseases. Malignant pleural effusion is a common problem in cancer patients. It usually occurs in advanced cancer patients with short life expectancy. ${ }^{19}$ When a patient with suspected MPM has pleural effusion, 
the diagnosis should begin with an ultrasound-guided thoracentesis, and the pleural effusion should be sent for cytopathology analysis. Although less than one third of MPM can accurately diagnose pleural fluid cytology. Sarcomatoid and biphasic mesothelioma are rarely detected in pleural fluid specimens. ${ }^{20}$ In addition, pleural fluid cytology is difficult in the diagnosis and classification of benign and malignant tumors, and is not the first choice for MPM diagnosis. Thoracoscopy and pleural biopsy are usually recommended to obtain tissue specimens to diagnose MPM and determine histological MPM subtypes. If thoracoscopy is not feasible, CT-guided pleural biopsy or open pleural biopsy is recommended as the next best diagnostic method. ${ }^{20}$ In case of negative CT-guided pleural biopsies, VATS under local anesthesia for diagnosis could have been performed in frail patients also with. ${ }^{17,18}$ In addition, PET-CT can not only evaluate the whole body of the lesion, but also visually display the most active metabolic site of pleural lesion. 18F-FDG uptake and/or the most accessible sites can be identified and targeted for tissue sampling. Whenever possible, PET-CT examination should be combined with histological biopsy to confirm the diagnose of tumor. ${ }^{21}$ In our case, because the open pleural biopsies for the patient was not feasible, we performed a PET-CT examination. The results suggested that the left pleurais thickened and calcified. FDG metabolism increased, and malignant tumor was considered. A tissue biopsy was then performed on the metabolically active lesions to confirm the diagnosis.

Tissue biopsy specimens require pathological and immunohistochemical tests to confirm diagnosis and histological subtypes. Sarcomatoid MPM is composed of malignant spindle cells growing in fascicles or randomly in fibrous stroma. Then, our patient's pathology showed that the tumor was composed of fibroblast-like spindle cells arranged in bundles or chaotically. The tumor cells had obvious atypia, easy to see mitotic figures and coagulative necrosis, which is more consistent with pathological manifestations of sarcomatoid mesothelioma. And the immunohistochemistry revealed that WT1, CK (Pan) and GATA3 were positive, and P63, TTF-1, EMA, Calretinin, CK5/6, D2-40, Vimentin, Desmin, CEA were all negative. Generally speaking, sarcomatoid MPM does not express traditional mesothelioma markers such as CK5/6, D2-40 and calretinin, but only pan-cytokeratin (pan-CK). ${ }^{22}$ Sarcomatoid malignant mesotheliomas is usually stained for pan-CKs with antibodies AE1/AE3. The study of Lucas et $\mathrm{al}^{23}$ showed that $70 \%$ of sarcomatoid malignant mesotheliomas were stained with pan-CK. The immunohistochemistry in this report showed that CK (Pan) was positive, and Calretinin, CK5/6, D2-40 were all negative, which was consistent with the above results. In addition, immunohistochemistry showed positive for WT1 and GATA3. Wilms tumor-1 protein (WT1) is highly overexpressed in MPM, but is particularly low in lung adenocarcinoma. Pathologists routinely use WT1 immunohistochemical (IHC) expression for pathologic diagnosis of MPM. The WT1 is expressed in approximately $97-98 \%$ of both epithelioid and non-epithelioid MPM with limited normal expression elsewhere. ${ }^{24,25}$ In 2017, Berg et al performed GATA binding protein 3 (GATA3) staining on 19 patients with sarcomatoid malignant mesothelioma and 13 patients with sarcomatoid lung cancer. The results indicated that all 19 cases of sarcomatoid malignant mesothelioma showed strong diffuse staining for GATA3, while only 2 cases of pulmonary sarcomatoid carcinoma showed positive staining for GATA3, and the staining was weak and inconsistent. ${ }^{26}$ Therefore, compared with sarcomatoid carcinoma of the lung, the GATA3 staining is more helpful in the diagnosis of sarcomatoid malignant mesothelioma. Moreover, P63 staining is positive in almost all squamous cell carcinomas, negative in mesothelioma, and positive in a large proportion of lung adenocarcinomas. ${ }^{27} \mathrm{CEA}$ and TTF-1 are considered as markers of lung adenocarcinoma. The patient's immunohistochemistry showed that CEA, p63 and TTF-1 were negative, which effectively ruled out the possibility of diagnosis of lung adenocarcinoma. In our case, the final diagnosis of sarcomatoid MPM was mainly based on clinical manifestations, imaging, pathology and immunohistochemistry results. The diagnosis rate of MPM needs to be improved.Especially, some rare types of mesothelioma, for example sarcomatoid MPM. Malignant mesothelioma is fatal. Early and accurate diagnosis can greatly improve the prognosis.

The treatment of MPM can be roughly divided into palliative treatment and curative (or radical) treatment. Palliative strategies are more likely to be used for the elderly and frail people who have advanced cancer that cannot be controlled by other therapies. After a randomized trial was reported in $2003,{ }^{28}$ the combination of cisplatin/carboplatin and pemetrexed has been regarded as the standard systemic treatment of MPM. In addition, radiotherapy, molecular targeted therapy, immunotherapy novel antiangiogenic agents and pleurodesis also have certain clinical applications. ${ }^{29}$ The main radical strategy is surgical resection, including extrapleural pneumonectomy 
(EPP) and pleural resection/peeling, ${ }^{30}$ which can be performed before or after chemotherapy, radiotherapy, or both. After the publication of the randomized controlled trials on $\mathrm{EPP}^{31}$ the number of EPP decreased dramatically worldwide in favour of less invasive procedure such as pleurectomy/decortication. In addition, some studies have reported triple therapy, including induction chemotherapy, EPP and subsequent hemithoracic radiotherapy. In a non-randomized study, patients had a median survival time of up to 29 months, and a systematic review suggested that triple therapy may be beneficial to selected patients. ${ }^{32-34}$

\section{Conclusions}

In summary, this case shows that if the effusion does not resolve when the suspected cause has been treated further work is required. For the diagnosis of sarcomatoid MPM, pleural biopsy guided by PET-CT is a good choice for patients who cannot tolerate open pleural biopsy or medical thoracoscopy.

\section{Ethics}

This patient provided written informed consent for the publication of the case details and images. And ethical approval for this study was obtained from the meeting of ethics committee of Zhejiang Provincial People's Hospital.

\section{Funding}

This study was supported by Medical Science and Technology Project of Zhejiang province (No. 2019RC119), (No.2020KY432), (No.2020KY400) and (No.2021KY441), Research Project of Public Welfare Technology Application of Zhejiang Province Science and Technology Department (No. LGF20H010008), Zhejiang Natural ScienceFoundation (Q18H010007).

\section{Disclosure}

The authors report no conflicts of interest in this work.

\section{References}

1. Beckett P, Edwards J, Fennell D, Hubbard R, Woolhouse I, Peake MD. Demographics, management and survival of patients with malignant pleural mesothelioma in the National Lung Cancer Audit in England and Wales. Lung Cancer. 2015;88(3):344-348. doi:10.1016/j.lungcan. 2015.03.005

2. Soeberg MJ, Leigh J, van Zandwijk N. Malignant mesothelioma in Australia 2015: current incidence and asbestos exposure trends. $J$ Toxicol Environ Health B Crit Rev. 2016;19(5-6):173-189. doi:10. 1080/10937404.2016.1194254
3. Scherpereel A, Astoul P, Baas P, et al. [Guidelines of the European Respiratory Society and the European Society of Thoracic Surgeons for the management of malignant pleural mesothelioma]. Zhongguo Fei Ai Za Zhi = Chinese J Lung Cancer. 2010;13(10):C23-45. [Chinese]. doi:10.3779/j.issn.1009-3419.2010.10.14

4. Kakkar AK, Levine M, Pinedo HM, Wolff R, Wong J. Venous thrombosis in cancer patients: insights from the FRONTLINE survey. Oncologist. 2003;8(4):381-388. doi:10.1634/theoncologist.8-4-381

5. Rudd RM. Malignant mesothelioma. Br Med Bull. 2010;93:105-123. doi:10.1093/bmb/ldp047

6. Mazurek JM, Syamlal G, Wood JM, Hendricks SA, Weston A. Malignant mesothelioma mortality - United States, 1999-2015. MMWR Morb Mortal Wkly Rep. 2017;66(8):214-218. doi:10.15585/ mmwr.mm6608a3

7. Lacourt A, Gramond C, Rolland P, et al. Occupational and non-occupational attributable risk of asbestos exposure for malignant pleural mesothelioma. Thorax. 2014;69(6):532-539. doi:10.1136/ thoraxjnl-2013-203744

8. Astoul P, Roca E, Galateau-Salle F, Scherpereel A. Malignant pleural mesothelioma: from the bench to the bedside. Respiration. 2012;83 (6):481-493. doi:10.1159/000339259

9. Guo Z, Carbone M, Zhang X, et al. Improving the accuracy of mesothelioma diagnosis in China. $J$ Thorac Oncol. 2017;12 (4):714-723. doi:10.1016/j.jtho.2016.12.006

10. British Thoracic Society Standards of Care Committee. BTS statement on malignant mesothelioma in the UK, 2007. Thorax. 2007;62 (Suppl 2):ii1-ii19. doi:10.1136/thx.2007.087619

11. Scherpereel A, Astoul P, Baas P, et al. Guidelines of the European Respiratory Society and the European Society of Thoracic Surgeons for the management of malignant pleural mesothelioma. Eur Respir J. 2010;35(3):479-495. doi:10.1183/09031936.00063109

12. Marchevsky AM, LeStang N, Hiroshima K, et al. The differential diagnosis between pleural sarcomatoid mesothelioma and spindle cell/pleomorphic (sarcomatoid) carcinomas of the lung: evidence-based guidelines from the International Mesothelioma Panel and the MESOPATH National Reference Center. Hum Pathol. 2017;67:160-168. doi:10.1016/j.humpath.2017.07.015

13. Liu M, Cui A, Zhai ZG, et al. Incidence of pleural effusion in patients with pulmonary embolism. Chin Med J. 2015;128(8):1032-1036. doi:10.4103/0366-6999.155073

14. Koksal D, Safak O, Ozcan A, Agackiran Y, Erturk H, Findik G. Thromboembolic events in malignant pleural mesothelioma. Clin Appl Thromb Hemost. 2016;22(4):390-394. doi:10.1177/1076029614564861

15. Nakano T, Chahinian AP, Shinjo M, et al. Interleukin 6 and its relationship to clinical parameters in patients with malignant pleural mesothelioma. $\mathrm{Br}$ J Cancer. 1998;77(6):907-912. doi:10.1038/bjc.1998.150

16. Idell S, Pueblitz S, Emri S, et al. Regulation of fibrin deposition by malignant mesothelioma. Am J Pathol. 1995;147(5):1318-1329.

17. Migliore M. Efficacy and safety of single-trocar technique for minimally invasive surgery of the chest in the treatment of noncomplex pleural disease. J Thorac Cardiovasc Surg. 2003;126(5):1618-1623. doi:10.1016/S0022-5223(03)00592-0

18. Migliore M, Giuliano R, Aziz T, Saad RA, Sgalambro F. Four-step local anesthesia and sedation for thoracoscopic diagnosis and management of pleural diseases. Chest. 2002;121(6):2032-2035. doi:10.1378/chest.121.6.2032

19. Froudarakis ME. Diagnostic work-up of pleural effusions. Respiration. 2008;75(1):4-13. doi:10.1159/000112221

20. Kindler HL, Ismaila N, Armato SG, et al. Treatment of malignant pleural mesothelioma: american society of clinical oncology clinical practice guideline. J Clin Oncol. 2018;36(13):1343-1373. doi:10. 1200/JCO.2017.76.6394

21. Kitajima K, Doi H, Kuribayashi K. Present and future roles of FDG-PET/CT imaging in the management of malignant pleural mesothelioma. Jpn J Radiol. 2016;34(8):537-547. doi:10.1007/ s11604-016-0555-1 
22. Klebe S, Brownlee NA, Mahar A, et al. Sarcomatoid mesothelioma: a clinical-pathologic correlation of 326 cases. Mod Pathol. 2010;23 (3):470-479. doi:10.1038/modpathol.2009.180

23. Lucas DR, Pass HI, Madan SK, et al. Sarcomatoid mesothelioma and its histological mimics: a comparative immunohistochemical study. Histopathology. 2003;42(3):270-279. doi:10.1046/j.1365-2559.2003. 01583.x

24. Eguchi T, Kadota K, Mayor M, et al. Cancer antigen profiling for malignant pleural mesothelioma immunotherapy: expression and coexpression of mesothelin, cancer antigen 125, and Wilms tumor 1. Oncotarget. 2017;8(44):77872-77882. doi:10.18632/ oncotarget. 20845

25. Zauderer MG, Tsao AS, Dao T, et al. A randomized phase II trial of adjuvant galinpepimut-S, WT-1 analogue peptide vaccine, after multimodality therapy for patients with malignant pleural mesothelioma. Clin Cancer Res. 2017;23(24):7483-7489. doi:10.1158/1078-0432. CCR-17-2169

26. Berg KB, Churg A. GATA3 immunohistochemistry for distinguishing sarcomatoid and desmoplastic mesothelioma from sarcomatoid carcinoma of the lung. Am J Surg Pathol. 2017;41(9):1221-1225. doi:10. 1097/PAS.0000000000000825

27. Kriegsmann K, Cremer M, Zgorzelski C, et al. Agreement of CK5/6, p40, and p63 immunoreactivity in non-small cell lung cancer. Pathology. 2019;51(3):240-245. doi:10.1016/j.pathol.2018.11.009

28. Vogelzang NJ, Rusthoven JJ, Symanowski J, et al. Phase III study of pemetrexed in combination with cisplatin versus cisplatin alone in patients with malignant pleural mesothelioma. J Clin Oncol. 2003;21 (14):2636-2644. doi:10.1200/JCO.2003.11.136-8
29. Wald O, Sugarbaker DJ. New concepts in the treatment of malignant pleural mesothelioma. Annu Rev Med. 2018;69:365-377. doi:10. 1146/annurev-med-041316-085813

30. Bertoglio P, Waller DA. The role of thoracic surgery in the management of mesothelioma: an expert opinion on the limited evidence. Expert Rev Respir Med. 2016;10(6):663-672. doi:10.1586/1747 6348.2016.1171147

31. Treasure T, Lang-Lazdunski L, Waller D, et al. Extra-pleural pneumonectomy versus no extra-pleural pneumonectomy for patients with malignant pleural mesothelioma: clinical outcomes of the Mesothelioma and Radical Surgery (MARS) randomised feasibility study. Lancet Oncol. 2011;12 (8):763-772. doi:10.1016/S1470-2045(11)70149-8

32. Van Schil PE, Baas P, Gaafar R, et al. Trimodality therapy for malignant pleural mesothelioma: results from an EORTC phase II multicentre trial. Eur Respir J. 2010;36(6):1362-1369. doi:10.1183/ 09031936.00039510

33. Cao C, Tian D, Manganas C, Matthews P, Yan TD. Systematic review of trimodality therapy for patients with malignant pleural mesothelioma. Ann Cardiothorac Surg. 2012;1(4):428-437. doi:10. 3978/j.issn.2225-319X.2012.11.07

34. Krug LM, Pass HI, Rusch VW, et al. Multicenter phase II trial of neoadjuvant pemetrexed plus cisplatin followed by extrapleural pneumonectomy and radiation for malignant pleural mesothelioma. J Clin Oncol. 2009;27(18):3007-3013. doi:10.1200/JCO.2008.20.3943
OncoTargets and Therapy

\section{Publish your work in this journal}

OncoTargets and Therapy is an international, peer-reviewed, open access journal focusing on the pathological basis of all cancers, potential targets for therapy and treatment protocols employed to improve the management of cancer patients. The journal also focuses on the impact of management programs and new therapeutic agents and protocols on patient perspectives such as quality of life, adherence and satisfaction. The manuscript management system is completely online and includes a very quick and fair peer-review system, which is all easy to use. Visit http://www.dovepress.com/ testimonials.php to read real quotes from published authors. 